\title{
Errata to "Tidal friction in close-in satellites and exoplanets. The Darwin theory re-visited"
}

\author{
Sylvio Ferraz-Mello • Adrián Rodríguez • \\ Hauke Hussmann
}

Published online: 16 April 2009

(C) Springer Science+Business Media B.V. 2009

\section{Erratum to: Celest Mech Dyn Astr: 101, 171-201 DOI 10.1007/s10569-008-9133-x}

Correction of misprints in the equations giving the cumulative orbital variations in the orbital elements of an exoplanet due to tides in both star and planet. Correction of the equations of variation in the argument of perihelion. Variation of the node in the same approximation.

\section{Equations 92 and 93}

Misprints were found in Eqs. 92 and 93. The right equations are:

$$
\begin{aligned}
& <\dot{n}>=\frac{9 n^{2} k_{d A} m_{B} R_{A}^{5}\left|\varepsilon_{0 A}^{\prime}\right|}{2 m_{A} a^{5}}\left(1+23 e^{2}+D\left(7 e^{2}+S_{B}^{2}\right)+\frac{\Omega_{A}}{2 n}\left(19 e^{2}+S_{A}^{2}\right)\right) \\
& <\dot{e}>=-\frac{27 n e k_{d A} m_{B} R_{A}^{5}\left|\varepsilon_{0 A}^{\prime}\right|}{2 m_{A} a^{5}}\left(1+\frac{7}{9} D+\frac{7}{18} \frac{\Omega_{A}}{n}\right)
\end{aligned}
$$

\section{Appendix B: Variations in the argument of pericenter and node}

The results are incomplete. To obtain the variations up to second-degree in $e, I$, it is necessary to consider in $U_{2}$ terms up to fourth degree and

$$
\varphi \simeq v+\omega-\frac{1}{4} \sin (2 v+2 \omega) \sin ^{2} I-\left(\frac{1}{8} \sin (2 \omega+2 v)-\frac{1}{32} \sin (4 \omega+4 v)\right) \sin ^{4} I
$$

The online version of the original article can be found under doi:10.1007/s10569-008-9133-x.

S. Ferraz-Mello $(\varangle) \cdot$ A. Rodríguez

Instituto de Astronomia, Geofísica e Ciências Atmosféricas, Universidade de São Paulo,

São Paulo, SP, Brazil

e-mail: sylvio@astro.iag.usp.br

H. Hussmann

Institut für Planetenforschung, DLR, Berlin-Adlershof, Germany 
instead of the approximation given in Eq. 8 .

The corrected second-degree results are

$$
\begin{aligned}
<\dot{\omega}_{\text {lag }}>= & \frac{9 k_{d} n M R^{5}}{16 m a^{5}}\left[\left(3 \varepsilon_{0}^{\prime}-\varepsilon_{2}^{\prime}-\varepsilon_{5}^{\prime}+3 \varepsilon_{6}^{\prime}-3 \varepsilon_{8}^{\prime}-\varepsilon_{15}^{\prime}+\varepsilon_{16}^{\prime}+3 \varepsilon_{25}^{\prime}\right) e^{2}\right. \\
& \left.-\frac{1}{2}\left(6 \varepsilon_{0}^{\prime}-\varepsilon_{2}^{\prime}-\varepsilon_{5}^{\prime}-6 \varepsilon_{7}^{\prime}-12 \varepsilon_{8}^{\prime}+\varepsilon_{11}^{\prime}+\varepsilon_{12}^{\prime}-2 \varepsilon_{15}^{\prime}+2 \varepsilon_{16}^{\prime}\right) S^{2}\right] \sin 2 \omega
\end{aligned}
$$

and

$$
<\dot{\omega}^{U}>=\frac{15 k_{f} n M R^{5}}{2 m a^{5}}\left(1+\frac{13}{2} e^{2}\right) .
$$

At the same approximation, the tidal variation of the node is given by

$$
<\widehat{\mathrm{ON}}_{\mathrm{lag}}>=-\frac{9 k_{d} n M R^{5}}{16 m a^{5}}\left(3 \varepsilon_{0}^{\prime}-\varepsilon_{2}^{\prime}-\varepsilon_{5}^{\prime}+3 \varepsilon_{6}^{\prime}-3 \varepsilon_{8}^{\prime}-\varepsilon_{15}^{\prime}+\varepsilon_{16}^{\prime}+3 \varepsilon_{25}^{\prime}\right) e^{2} \sin 2 \omega \text {. }
$$

\section{Equation 24}

It is also necessary to consider third-degree terms in $U_{\text {lag }}$ to complete Eq. 24 . The missing terms, however, do not affect the results given in the paper (except in Appendix B). The complete second-degree expression of $F_{2}$ is:

$$
\begin{aligned}
F_{2}(\mathbf{r})= & \frac{3 k_{d} G M^{2} R^{5}}{8 a^{7}}\left[e S\left(8 \varepsilon_{0}^{\prime}-7 \varepsilon_{1}^{\prime}-\varepsilon_{2}^{\prime}+6 \varepsilon_{5}^{\prime}-12 \varepsilon_{8}^{\prime}+4 \varepsilon_{9}^{\prime}+6 \varepsilon_{15}^{\prime}+2 \varepsilon_{16}^{\prime}\right) \cos \omega\right. \\
& -e S\left(8 \varepsilon_{0}^{\prime}-7 \varepsilon_{1}^{\prime}-\varepsilon_{2}^{\prime}+6 \varepsilon_{5}^{\prime}+4 \varepsilon_{8}^{\prime}-12 \varepsilon_{9}^{\prime}-6 \varepsilon_{14}^{\prime}+14 \varepsilon_{17}^{\prime}\right) \cos (2 \ell+\omega) \\
& \left.-4 S\left(\varepsilon_{8}^{\prime}-\varepsilon_{9}^{\prime}\right) \cos (\ell+\omega)\right] .
\end{aligned}
$$

With this correction, Eqs. 22-24 are equivalent to the second-degree expansion of the components of the force given by Mignard (1979).

\section{New frequencies}

The frequencies of the new phase lags appearing in the above equations are given below:

\begin{tabular}{ll}
\hline Subscript no. & Frequency \\
\hline 11 & $2 \Omega-n$ \\
12 & $n$ \\
14 & $\Omega+n$ \\
15,16 & $\Omega-n$ \\
17 & $\Omega-3 n$ \\
25 & $\Omega-2 n$ \\
\hline
\end{tabular}

\section{References}

Ferraz-Mello, S., Rodríguez, A., Hussmann, H.: Tidal friction in close-in satellites and exoplanets: The Darwin theory re-visited. ArXiv: 0712.1156 (astro-ph)

Mignard, F.: The evolution of the lunar orbit revisited-I. Moon Planets 20, 301-315 (1979) 